\title{
On the Boundary Layer Flow over a Moving Surface in a Fluid with Temperature-Dependent Viscosity
}

\author{
E. W. Mureithi ${ }^{1}$, J. J. Mwaonanji ${ }^{1}$, O. D. Makinde ${ }^{2}$ \\ ${ }^{1}$ Department of Mathematics, University of Dar es Salaam, Dar es Salaam, Tanzania \\ ${ }^{2}$ Cape Peninsula University of Technology, Bellville, South Africa \\ Email: ewambui02@gmail.com
}

Received March 25, 2013; revised April 28, 2013; accepted May 6, 2013

Copyright (C) 2013 E. W. Mureithi et al. This is an open access article distributed under the Creative Commons Attribution License, which permits unrestricted use, distribution, and reproduction in any medium, provided the original work is properly cited.

\begin{abstract}
This paper examines a boundary layer flow over a continuously moving heated flat surface with velocity $U_{\omega}(x)$ in a streaming flow with velocity $U_{\infty}(x)$ and with temperature dependent viscosity, $\mu(T)$. The momentum and the energy equations are coupled through the viscous dissipation term. The coupled boundary layer equations are transformed into a self-similar form using an appropriate similarity variable. An efficient numerical technique is used to solve the self-similar boundary layer equations. It is shown that at low enough values for the velocity ratio $\xi$, an increase in viscous dissipation enhances greatly the local heat transfer leading to temperature overshoots adjacent to the wall. The viscosity variation parameter is shown to have significant effects on the temperature dependent viscosity and the velocity and temperature distribution within the boundary layer.
\end{abstract}

Keywords: Flat Moving Surface; Temperature Dependent Viscosity; Self-Similar Equations; Viscous Dissipation

\section{Introduction}

Studies on heat and mass transfer in boundary layers over continuously moving or stretching surfaces have been increasing due to their wide variety of applications in manufacturing processes such as glass-fibre production, metal extrusion, materials-handling conveyors and paper production.

One of the earliest studies on boundary-layer flow past moving surfaces was initiated by Sakiadis [1], who investigated momentum transfer for a flow over a continuously moving plate in quiescent fluid. The results of Sakiadis were later verified experimentally by Tsou et al. [2]. Over recent years studies of boundary layer past moving or stretching surfaces in otherwise quiescent fluids included the work of Ali [3] who investigated similarity solutions for a thermal boundary layer over a power-law stretching surface with suction or injection; Elbashbeshy [4] who studied heat transfer over a stretching surface with suction or injection; Magyari and Keller [5] who studied similarity solutions for boundary layer flow over an exponentially stretching surface and Mureithi [6] who examined linear stability properties of a boundary layer flow over a moving surface in a streaming flow.

Studies on free-stream effects on boundary-layer flows over moving or stretching surfaces included the work of Abdelhafez [7] and Chappidi and Gunnerson [8] who independently considered flows over moving surfaces in which both the surface and the free stream moved in the same direction. In their studies, they formulated two sets of boundary value problems for the cases $U_{\infty}<U_{\omega}$ and $U_{\infty}>U_{\omega}$. Afzal [9] formulated a single set of equations using as reference velocity a composite velocity given by $\bar{U}=U_{\infty}+U_{\omega}$. Later Lin and Huang [10] used Afzal's formulation to study momentum and heat transfer for a flow over a surface moving parallel or reversely to the free stream with temperature dependent viscosity. A study by Afzal [11] investigated momentum transfer on a power law stretching surface with free-stream pressure gradient.

The current study investigates a boundary layer flow over a moving surface in a streaming flow with a temperature dependent dynamic viscosity, $\mu(T)$. The Ling and Dybbs [12] model for $\mu(T)$ is used in this study.

In Section 2, we formulate the problem. In Section 3, boundary layer equations are reduced to the self-similar form. In Section 4, numerical solutions for the self-similar boundary layer equations are presented and discussed and conclusions are drawn in Section 5. 


\section{Problem Formulation}

An incompressible flow past an infinite surface continuously moving with velocity $U_{\omega}$ in a streaming flow with velocity $U_{\infty}$ and with temperature dependent viscosity $\mu(T)$, is investigated. The fluid is of density $\rho$, thermal conductivity $\kappa$ and specific heat capacity $c_{p}$ (at constant pressure). The boundary layer equations are

$$
\left.\begin{array}{l}
\frac{\partial u}{\partial x}+\frac{\partial v}{\partial y}=0 \\
\rho\left(u \frac{\partial u}{\partial x}+v \frac{\partial u}{\partial y}\right)=\rho U_{\infty} \frac{\mathrm{d} U_{\infty}}{\mathrm{d} x}+\frac{\partial}{\partial y}\left(\mu(T) \frac{\partial u}{\partial y}\right) \\
u \frac{\partial T}{\partial x}+v \frac{\partial T}{\partial y}=\frac{\kappa}{\rho c_{p}} \frac{\partial^{2} T}{\partial y^{2}}+\frac{\mu(T)}{\rho c_{p}}\left(\frac{\partial u}{\partial y}\right)^{2}
\end{array}\right\}
$$

The boundary conditions for this flow are

$$
\left.\begin{array}{l}
u(x, 0)=U_{\omega}(x), v(x, 0)=0, T(x, 0)=T_{\omega}, \\
u(x, \infty)=U_{\infty}(x), T(x, \infty)=T_{\infty} .
\end{array}\right\}
$$

for a flow over an impermeable surface $v(x, 0)=0$.

The following temperature dependent viscosity model due to Ling and Dybbs [12] is used here:

$$
\mu(T)=\frac{\mu_{\infty}}{1+\gamma\left(T-T_{\infty}\right)}
$$

where $\gamma$ is a constant, $\mu_{\infty}$ is the constant reference viscosity in the absence of heating. The case $\gamma=0$ corresponds to the constant viscosity situation.

\section{Self-Similar Boundary Layer Equations}

The basic flow is rendered in non-dimensional form through setting

$$
\begin{aligned}
& u(x, y)=\bar{U} f^{\prime}(\eta), T(x, y)-T_{\infty}=\left(T_{w}-T_{\infty}\right) \theta(\eta), \\
& \eta=\frac{y}{x} \operatorname{Re}_{x}^{1 / 2},
\end{aligned}
$$

where $\bar{U}(x)=U_{\omega}+U_{\infty}$ is the reference velocity (Afzal et al. [9]), $\eta(x, y)$ is the boundary-layer similarity variable and $f^{\prime}(\eta)$ and $\theta(\eta)$ are the scaled free-stream velocity and temperature, respectively. The parameter $R e_{x}$ is the local Reynolds number defined as $R e_{x}=\frac{\bar{U} x}{v}$.

In non-dimensional form, the Lings-Dybbs model becomes

$$
\frac{\mu}{\mu_{\infty}}=\frac{1}{1+\epsilon \theta}
$$

where $\mu / \mu_{\infty}$ is the dimensionless dynamic viscosity and $\epsilon=\gamma\left(T_{\omega}-T_{\infty}\right)$ is the variable viscosity parameter. The case $\epsilon=0$ is equivalent to the case $\gamma=0$ corresponding to constant viscosity.

From the equation of continuity we have

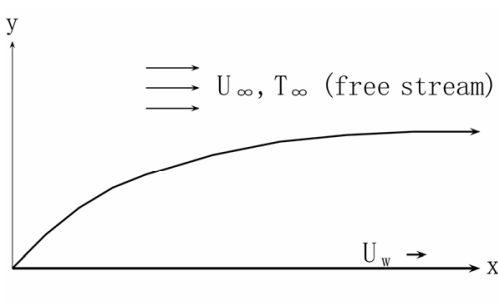

$\mathrm{u}=\mathrm{U}_{\mathrm{w}}(\mathrm{x}), \mathrm{v}=\mathrm{V}_{\mathrm{w}}, \mathrm{T}=\mathrm{T}_{\mathrm{w}}$

Figure 1. Schematic diagram for the problem.

$$
v(x, y)=-\frac{\bar{U}}{2 \operatorname{Re}_{x}^{1 / 2}}\left[(\beta+1) f+\eta(\beta-1) f^{\prime}\right], \beta=\frac{x}{\bar{U}} \frac{\mathrm{d} \bar{U}}{\mathrm{~d} x}
$$

where the parameter $\beta$ reduces to the pressure gradient parameter

$$
\beta_{0}=\frac{x}{U_{\infty}} \frac{\mathrm{d} U_{\infty}}{\mathrm{d} x} \text { if } U_{\omega}=0 .
$$

We assume power-law variations in the free-stream velocity and wall velocity of the form $U_{\omega}=U_{0} x^{n}, U_{\infty}=U_{e} x^{n}$ so that.

$$
\bar{U}=U_{\omega}(x)+U_{\infty}(x)=\left(U_{0}+U_{e}\right) x^{n}=A x^{n}
$$

and $\beta=\beta_{0}=n$. The dimensionless similarity boundarylayer equations take the form

$$
\begin{gathered}
f^{\prime \prime \prime}=(1+\epsilon \theta)\left[n\left(\left(f^{\prime}\right)^{2}-\xi^{2}\right)-\frac{n+1}{2} f f^{\prime \prime}\right]+\frac{\epsilon \theta^{\prime} f^{\prime \prime}}{1+\epsilon \theta}, \\
\frac{1}{P r} \theta^{\prime \prime}=-\frac{1}{2}(n+1) f \theta^{\prime}-\frac{E c x^{2 n}}{\xi^{2}(1+\epsilon \theta)}\left(f^{\prime \prime}\right)^{2} .
\end{gathered}
$$

with boundary conditions

$$
\left.\begin{array}{l}
f(0)=0, f^{\prime}(0)=1-\xi, \theta(0)=1, \\
f^{\prime} \rightarrow \xi, \theta \rightarrow 0 \text { as } \eta \rightarrow \infty,
\end{array}\right\}
$$

where

$$
\xi=\frac{U_{\infty}}{U_{\omega}+U_{\infty}}, \operatorname{Pr}=\frac{\mu c_{p}}{\kappa}, E c=\frac{U_{e}^{2}}{c_{p}\left(T_{\omega}-T_{\infty}\right)} .
$$

The parameter $\operatorname{Pr}$ is the Prandtl number and $E c$ is the Eckert number. The flow is self-similar if one of the following is satisfied:

1. $n=0$ for any $E c$.

2. $E c=0$ for any $n$ (negligible viscous dissipation). remarks

We have assumed that both the wall and the free stream move in the same direction so that $0<\xi<1$. The case when $\xi=0$ is corresponds to a wall moving in an otherwise quiescent fluid $\left(U_{\infty}=0\right), \xi=1$ corresponds to flow over a stationary wall $\left(U_{\omega}=0\right)$ and $\xi=1 / 2$ is equivalent to $U_{\omega}=U_{\infty}$ so that the wall and the freestream move with the same speed. When $0<\xi<1 / 2$, the wall moves faster that the free-stream while the case when $1 / 2<\xi<1$ corresponds to the free-stream moving faster than the wall.

The surface shear stress and surface heat transfer are 
represented using the local skin friction factor, $C_{f}$, and the local Nusselt number, $N u_{x}$, respectively defined as

$$
C_{f} R e_{x}^{1 / 2}=\frac{f^{\prime \prime}(0)}{\xi^{2}(1+\epsilon)}, N u_{x} R e_{x}^{-1 / 2}=-\theta^{\prime}(0) .
$$

\section{Numerical Solution and Discussion of Results}

The coupled self-similar boundary layer Equations (3) and (4) together with the boundary conditions (5) are solved numerically using a shooting method coupled with the fourth-order Runge-Kutta scheme.

The results presented here are for the cases when $\operatorname{Pr}=0.72$. Self-similar solutions were obtained for two cases. Case one is the flow viscous dissipation and $n=0$. Case two corresponds to the case with $n \neq 0$ but without viscous dissipation effects.

Figures 2 and $\mathbf{3}$ show that the effect of varying the fluid viscosity variation parameter $\epsilon$ on the temperaturedependent dynamic viscosity, $\mu / \mu_{\infty}$ and the streamwise velocity $f^{\prime}(\eta)$, within the boundary layer. At any location within the boundary layer $\mu / \mu_{\infty}$ decreases with increase in the viscosity parameter, $\epsilon$. The boundary layer thickness is found to decrease with increase in $\epsilon$. The parameter $\epsilon$ is a measure of fluid viscosity variation.

The effect of varying viscous dissipation parameter, $E c$, and the velocity ratio $\xi$ on the temperature distribution in the boundary layer is shown in Figures 4 and 5. Figure 4 shows that for $\xi=0.1$, increasing $E c$ results in temperature over-shoot near the wall, with peaks in creasing with increase in $E c$. Figure 5 illustrates the effect of varying the velocity ratio on the temperature distribution. For the case when $E c=0.5$, the results show that the temperature peaks are realized for $\xi<0.3$ and the peaks amplitudes increase with decrease in $\xi$.

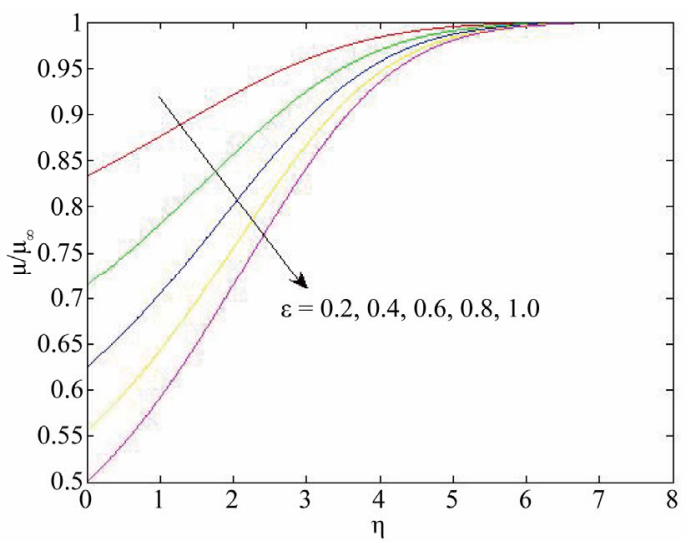

Figure 2. Effects of viscosity variation parameter on the dynamic viscosity for $n=0, \xi=0.8, E c=0.5, \epsilon=0.2,0.4,0.6,0.8,1$.

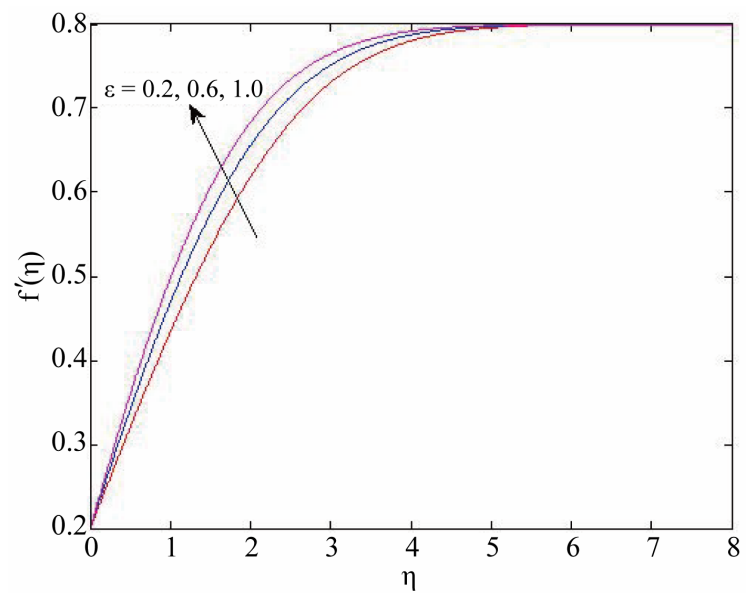

Figure 3. Effects of viscosity variation parameter on velocity profiles for

$n=0.0, \xi=0.8, E c=0.5, \epsilon=0.2,0.4,0.6,0.8,1$.

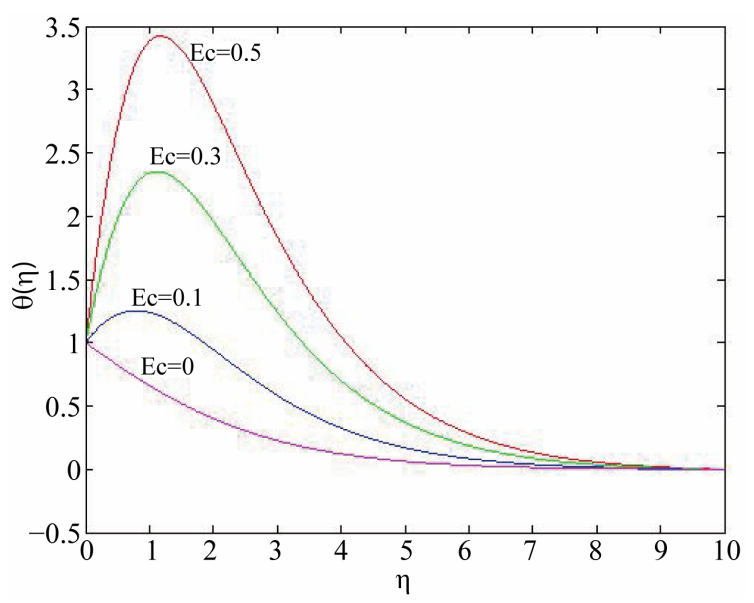

Figure 4. Effects of varying $E c$ on temperature distribution for $n=0.0, \xi=0.1, \epsilon=0.1, E c=0.1,0.3,0.5$.

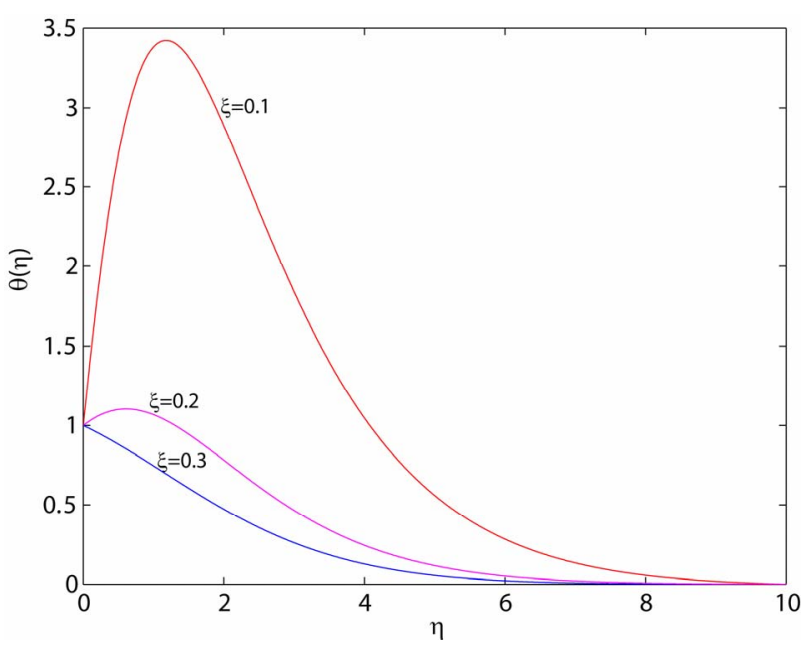

Figure 5. Effects of viscosity variation on the temperature distribution profiles for

$n=0.0, \epsilon=0.1, \xi=0.1,0.2,0.3, E c=0.5$. 
The skin-friction is presented as a function of $\epsilon$ in Figures 6 and 7. These figures show the effect of varying $\xi$ on the local skin friction coefficient for the case when $0.5<\xi<1$ and $0 \leq \xi<0.5$. For the case when

$0.5<\xi<1$, the local skin friction coefficient is positive and hence the fluid exerts a dragging force on the wall. For this case, increasing $\epsilon$ results in increase in the skin friction coefficient. For the case when $0 \leq \xi<0.5$, the local skin friction coefficient is negative, which is an indication that the wall drags the fluid. Also, increasing $\epsilon$ results in a decrease in the skin friction.

In Figure 8, the effect of varying $\xi$ on local Nusselt number is shown as a function of $\epsilon$ for the cases when $0.5<\xi<1$. The results show that the heat transfer coefficient decreases with increase in $\xi$ and increases with increase in $\epsilon$.

The results shown in Figure 9 are interesting. It is shown that for $0<\xi<0.3$, the local Nusselt number is

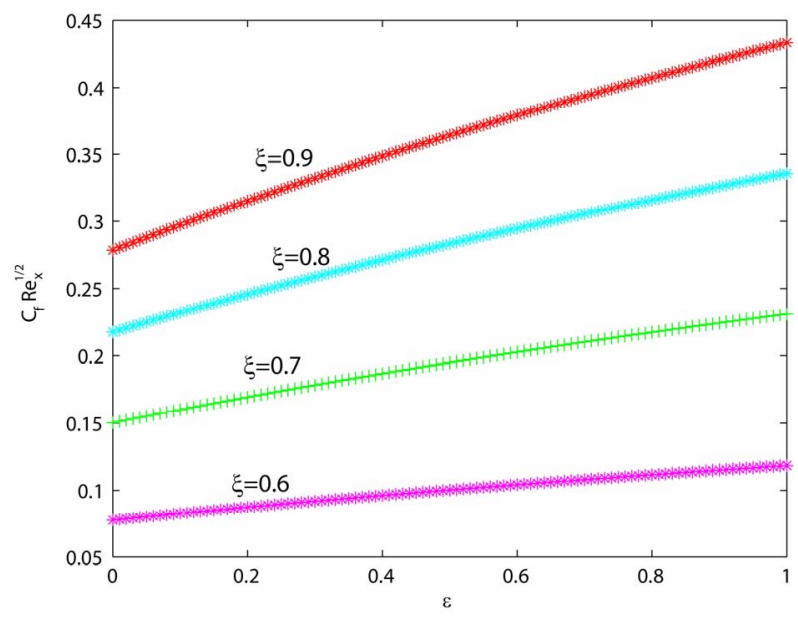

Figure 6. The skin friction coefficient against $\epsilon$ for the case when $n=0.0, E c=0.5, \xi=0.6,0.7,0.8,0.9$.

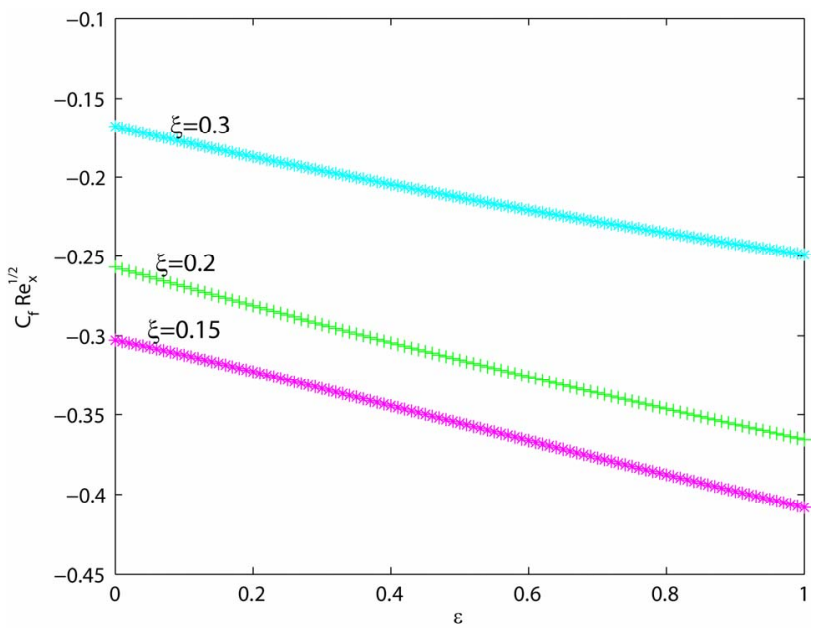

Figure 7. The skin friction coefficient against $\epsilon$ for the case when $n=0, E c=0.5, \xi=0.15,0.2,0.3$.

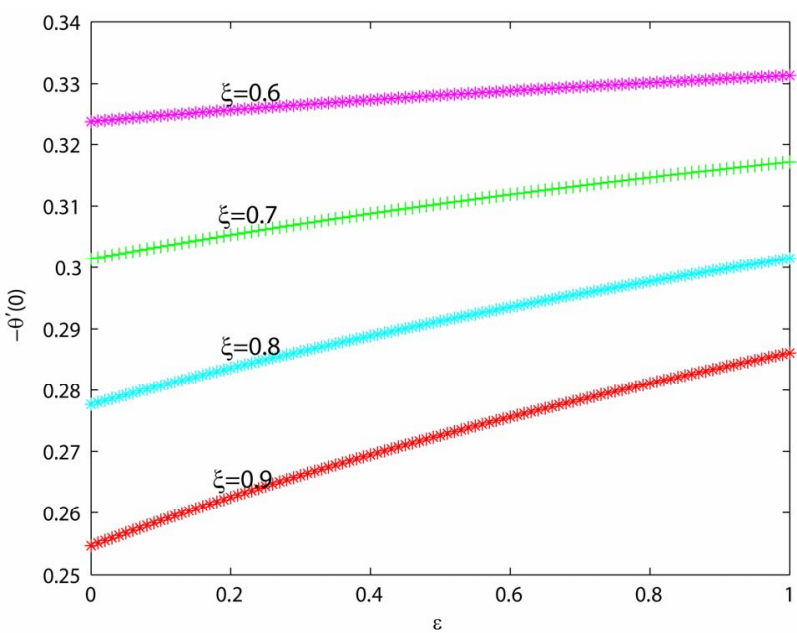

Figure 8. The heat transfer coefficient against $\epsilon$ for the case when $n=0, E c=0.5, \xi=0.6,0.7,0.8,0.9$.

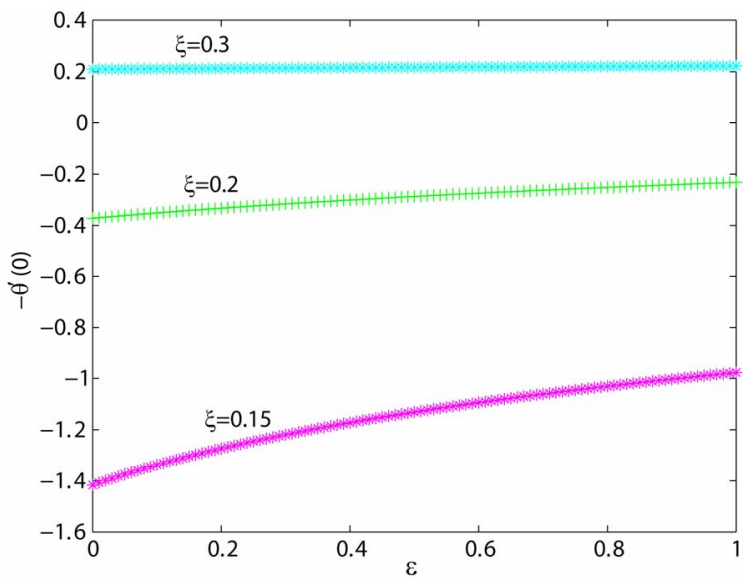

Figure 9. The heat transfer coefficient against $\epsilon$ for the case when $n=0, E c=0.5, \xi=0.15,0.2,0.25,0.3$.

negative, changing sign to positive for $\xi \geq 0.3$. This explains the results observed in Figure 7 where temperature over-shoot were observed adjacent to the wall. These results show that for low enough values for $\xi$, the heat transfer from wall to the fluid is greatly enhanced resulting in temperature over-shoots adjacent to the wall.

The effect of varying $\epsilon$ on the velocity and temperature distribution within the boundary layer is presented in Figures $\mathbf{1 0}$ and $\mathbf{1 1}$ for the case when wall is moving faster than the free-stream. The results show that velocity boundary layer thickness decreases with increase $\epsilon$ and that the temperature peaks decrease with increase in $\epsilon$. This shows that the increasing $\epsilon$ results in a decrease in heat transfer from the wall to the fluid.

\section{Conclusions}

A self-similar boundary layer flow has been presented 


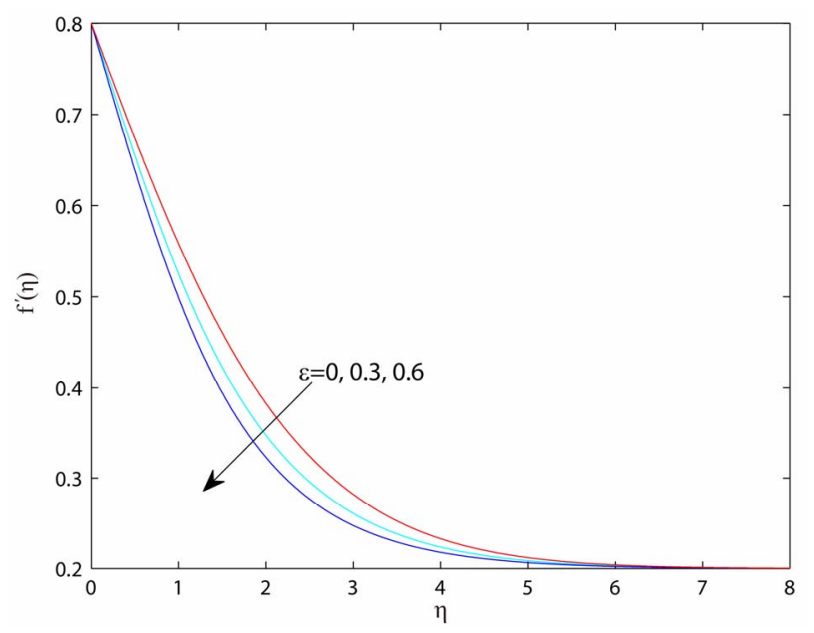

Figure 10. Effects of varying $\epsilon$ on the velocity distribution $f^{\prime}(\eta)$ for $n=0.0, \xi=0.2, E c=0.5$ and $\epsilon=0,0.3,0.6$.

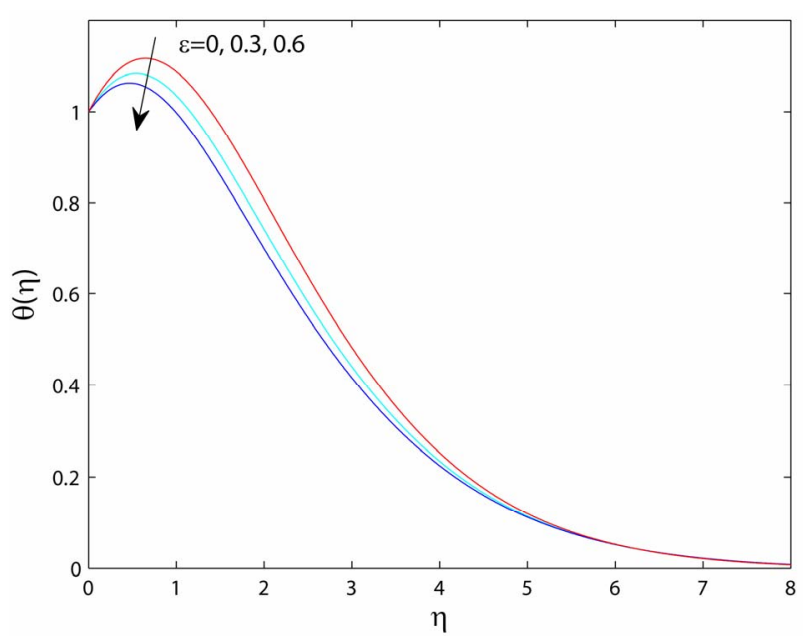

Figure 11. Effects of varying $\epsilon$ on the temperature distribution $\theta(\eta)$ for $n=0.0, \xi=0.2, E c=0.5$ and $\epsilon=0,0.3$,

0.6.

for a flow over a continuously moving heated surface in a fluid with temperature dependent viscosity. The selfsimilar equations were solved numerically and the results are presented in graphs.

In this study the effects of varying the viscosity variation $\epsilon$ and the velocity ratio $\xi$ are investigated for the case when the surface moves in the same direction as the free-stream.

For low enough values for the velocity ratio $\xi$, the local heat transfer is found to be negative, indicating the heat transfer from the wall to the fluid is greatly enhanced near the wall as the Eckert number increases. This is seen in the temperature distribution profiles where temperature peaks are observed adjacent to the wall. For the case when the wall moves faster than the fluid, the skin friction coefficient is negative, indicating that wall drags the fluid. The reverse occurs for the case when $0.5<\xi<1$ where the skin friction is positive and hence the free-stream exerts a dragging force on the boundary layer.

\section{REFERENCES}

[1] B. C. Sakiadis, "Boundary Layer Behavior on Continuous Solid Surfaces: II Boundary Layer on a Continuous Flat Surface," American Institute of Chemical Engineers Journal, Vol. 7, No. 2, 1961, pp. 221-225. doi:10.1002/aic.690070211

[2] F. Tsou, E. M. Sparrow and J. R. Goldstein, "Flow and Heat Transfer in the Boundary Layer on a Continuous Moving Surface," International Journal of Heat and Mass Transfer, Vol. 10, No. 2, 1967, pp. 219-235. doi:10.1016/0017-9310(67)90100-7

[3] M. E. Ali, "On the Thermal Boundary Layer on a PowerLaw Stretched Surface with Suction or Injection,” International Journal of Heat and Fluid Flow, Vol. 16, No. 4, 1995, pp. 280-290. doi:10.1016/0142-727X(95)00001-7

[4] E. M. Elbashbeshy, "Heat Transfer over a Stretching Surface with Variable Surface Heat Flux," Journal of Physics D: Applied Physics, Vol. 31, No. 16, 1998, pp. 19511954. doi:10.1088/0022-3727/31/16/002

[5] E. Magyari and B. Keller, "Heat and Mass Transfer in the Boundary Layer on an Exponentially Stretching Continuous Surface," Journal of Physics D: Applied Physics, Vol. 32, No. 5, 1999, pp. 577-585. doi:10.1088/0022-3727/32/5/012

[6] E. W. Mureithi, "Stability Properties of a Boundary Layer Flow Past a Continuously Moving Wall in a Streaming Flow," International Journal of Fluid Mechanics Research, Vol. 33, No. 5, 2006, pp. 430-444. doi:10.1615/InterJFluidMechRes.v33.i5.40

[7] T. A. Abdelhafez, "Skin Friction and Heat Transfer on a Continuous Flat Surface Moving in a Parallel Free Stream," International Journal of Heat and Mass Transfer, Vol. 28, No. 6, 1985, pp. 1234-1237. doi:10.1016/0017-9310(85)90132-2

[8] P. R. Chappidi and F. S. Gunnerson, "Analysis of Heat and Momentum Transport along a Moving Surface," International Journal of Heat and Mass Transfer, Vol. 32, No. 7, 1989, pp. 1383-1386. doi:10.1016/0017-9310(89)90039-2

[9] N. Afzal, A. Badaruddin and A. A. Elgarvi, "Momentum and Heat Transport on a Continuous Flat Surface Moving in a Parallel Stream," International Journal of Heat and Mass Transfer, Vol. 36, No. 13, 1993, pp. 3399-3403. doi:10.1016/0017-9310(93)90022-X

[10] H. T. Lin and S. F. Huang, "Flow and Heat Transfer of Plane Surface Moving in Parallel and Reversely to the Free Stream," International Journal of Heat and Mass Transfer, Vol. 37, No. 2, 1994, pp. 333-336. doi:10.1016/0017-9310(94)90104-X

[11] N. Afzal, "Momentum Transfer on Power Law Stretching Plate with Free Stream Pressure Gradient," International 
Journal of Engineering Science, Vol. 41, No. 11, 2003, pp. 1197-1207. doi:10.1016/S0020-7225(03)00002-8

[12] J. X. Lings and A. Dybbs, "Forced Convection over a Flat
Plate Submerged in a Porous Medium; Variable Viscosity Case,” ASME, New York, 1987. 